\title{
A Integral de Caminhos: Uma Ponte entre a Mecânica Quântica e a Mecânica Clássica
}

\author{
The Path Integral: A bridge between Quantum and Classical Mechanics \\ Alfredo M. Ozorio de Almeida*1 \\ ${ }^{1}$ Centro Brasileiro de Pesquisas Fisicas, Rio de Janeiro, RJ, Brazil
}

Recebido em 05 de Dezembro, 2017. Aceito em 15 de Fevereiro, 2018.

\begin{abstract}
A integral de caminhos de Feynman coloca de saída a ação clássica no cerne da evolução quântica. Ao invés de apenas contrastar a multiplicidade de caminhos quânticos com a raridade das trajetórias clássicas, podemos nos valer do princípio variacional que as identificam para construir abrangentes aproximações semiclássicas para o operador de evolução. A transformada de Fourier de seu traço fornece então a densidade de níveis quânticos de energia como uma soma sobre as órbitas periódicas clássicas: a fórmula do traço de Gutzwiller. As órbitas peródicas também explicam as correlações entre os níveis, seguindo padrões universais de matrizes aleatórias se o sistema clássico for caótico. Ao reverso, discrepâncias entre os espectros universais e os semiclássicos desvendaram correlações entre órbitas periódicas, nunca vislumbradas ao longo da história da mecânica clássica.

Palavras-chave: integral de caminho, trajetória clássica, princípio variacional, órbita periódica, densidade de níveis, matrizes aleatórias.

The Feynman path integral places the classical action in the very core of quantum evolution. Instead of contrasting the multiplicity of quantum paths with the scarcity of classical trajectories, we can avail ourselves of the variational principle by which they are identified. This leads to wide ranging semiclassical approximations for the evolution operator. The Fourier transform of the trace of this operator then supplies the density of quantum energy levels as a sum over the classical periodic orbits: The Gutzwiller trace formula. The periodic orbits also explain the correlations between levels, following universal classes of random matrices, if the classical system is chaotic. Conversely, discrepancies between the semiclassical spectra and the universal random matrix spectra uncouvered correlations between periodic orbits that had never been suspectected in the long history of classical mechanics.
\end{abstract}

Keywords: path integral, classical trajectory, variational principle, periodic orbit, level density, random matrices.

Ainda que as formulações de Heisenberg e de Schrödinger da mecânica quântica invoquem alguns elementos básicos da mecânica clássica, estes surgem de tal forma transfigurados que poderíamos bem duvidar que lidam com os mesmos sistemas. Afinal, a trajetória, solução das equações de Newton, Lagrange, ou Hamilton, personagem central da mecânica clássica, simplesmente some da mecânica quântica. Ou melhor, na reformulação de Feynman, ela retorna, mas apenas como um membro qualquer do coro, um dos múltiplos caminhos que contribuem democraticamente para o propagador. É na aproximação semiclássica que a trajetória readquire um certo destaque ao organizar a fase dos caminhos vizinhos de forma a diminuir radicalmente o número de contribuições ao propagador. A rica interação entre as mecânicas clássica e quântica que daí decorre mal poderia ser antevista quando da formulação da integral de caminhos, mas tem sobre esta uma base sólida.

*Endereço de correspondência: alfredozorio@gmail.com
É bom frisar que a relação entre a mecânica clássica e a mecânica quântica de que aqui tratamos é intrínsica à estrita evolucão unitária desta, pertinente aos sistemas dinâmicos fundamentais, isolados e conservativos: O sistema quântico inicial permanece quântico para todo e sempre. Aqui, as trajetórias clássicas tem papel de esqueleto sobre o qual são tecidas as ondas quânticas, tal qual os raios da ótica geométrica guiam as ondas luminosas. Daí, já podemos antecipar que as trajetórias terão na aproximação semiclássica da mecânica quântica uma participação mais coletiva do que na mecânica clássica. Nesse contexto, não há como pretender esclarecer qualquer aspecto da questão crucial de como características clássicas surgem gradualmente no processo de descoerência próprio de sistemas quânticos não isolados $[1,2$. Não é de surpreender que o próprio Feynman, por meio do desenvolvimento das integrais de trajetória conhecido como método de Feynman-Vernon 3, 4], desempenhou papel de destaque neste estudo, mas este será objeto de outros ensaios neste número especial. 
Consideremos um sistema de $L$ graus de liberdade, tal que os pontos, $\mathbf{x}=(\mathbf{p}, \mathbf{q})$, do espaço de fases clássico tem como coordenadas os $L$ momentos, $\mathbf{p}=\left(p_{1}, \ldots, p_{L}\right)$ e as $L$ posições, $\mathbf{q}=\left(q_{1}, \ldots, q_{L}\right)$. Na notação de Dirac, um auto-estado de posição, $\left|\mathbf{q}_{-}\right\rangle$evolui para o estado $|\mathbf{q}+(t)\rangle=\hat{U}_{t}\left|\mathbf{q}_{-}\right\rangle$, onde $\hat{U}_{t}=\mathrm{e}^{-i t \hat{H} / \hbar}$ é o operador de evolução e $\hat{H}$ é o operador hamiltoniano. Então, definimos o propagador, alvo da integral de trajetória, como $\left\langle\mathbf{q}_{+}\left|\hat{U}_{t}\right| \mathbf{q}_{-}\right\rangle=\left\langle\mathbf{q}_{+} \mid \mathbf{q}_{-}(t)\right\rangle$. O interessante é detectar uma dupla personalidade do propagador, pois, além de ser o overlap entre dois estados, podemos chegar a ele só por operadores: Sendo o operador de transição entre posições, $\left|\mathbf{q}_{-}\right\rangle\left\langle\mathbf{q}_{+}\right|$, seu traço com o operador de evolução, $\operatorname{tr} \hat{U}_{t}\left|\mathbf{q}_{-}\right\rangle\left\langle\mathbf{q}_{+}\right|=\left\langle\mathbf{q}_{+}\left|\hat{U}_{t}\right| \mathbf{q}_{-}\right\rangle$, ou seja, o propagador é também elemento de matriz do operador de evolução.

Existem várias formas de integrais de caminho, todas baseadas na idéia de Feynman [6] (antecipada por Dirac [5]) de aproveitar a propriedade de grupo do operador de evolução para avaliar

$$
\hat{U}_{t}=\lim _{N \rightarrow \infty}\left[\frac{\hat{U}_{t / N}}{N}\right]^{N} .
$$

As variações vem por conta da escolha de representação deste operador para tempos pequenos. Em vez de seguir o tratamento original de Feynman (esboçado também em [7]) vamos adotar aqui o de Marinov [8], pois, construida sobre caminhos no espaço de fases, se encaixa melhor nas considerações que se seguem. Em todo caso, pressupõe-se, como Feynman, uma Hamiltoniana da forma

$$
\hat{H}=H(\hat{\mathbf{p}}, \hat{\mathbf{q}})=\frac{\hat{\mathbf{p}}^{2}}{2 m}+V(\hat{\mathbf{q}})
$$

onde os operadores $\hat{\mathbf{p}}$ e $\hat{\mathbf{q}}$ tem $L$ componentes de momento e posição. Assim, podemos escrever a representação mista do operador de evolução para $t=\epsilon$ como

$$
\begin{aligned}
& \left\langle\mathbf{p}_{+}\left|\hat{U}_{\epsilon}\right| \mathbf{q}_{-}\right\rangle=\left\langle\mathbf{p}_{+}\left|\mathrm{e}^{-i \epsilon \hat{H} / \hbar}\right| \mathbf{q}_{-}\right\rangle \stackrel{\epsilon \rightarrow 0}{\longrightarrow} \\
& \left\langle\mathbf{p}_{+}\left|\mathrm{e}^{-i \epsilon \hat{\mathbf{p}}^{2} / 2 m \hbar} \mathrm{e}^{-i \epsilon V(\hat{\mathbf{q}}) / \hbar}\right| \mathbf{q}_{-}\right\rangle \\
& =\mathrm{e}^{-\frac{i \epsilon}{\hbar}\left[\frac{\mathbf{p}_{+}{ }^{2}}{2 m}+V\left(\mathbf{q}_{-}\right)\right]}\left\langle\mathbf{p}_{+} \mid \mathbf{q}_{-}\right\rangle,
\end{aligned}
$$

onde $\left\langle\mathbf{p}_{+} \mid \mathbf{q}_{-}\right\rangle \propto \mathrm{e}^{-\frac{i}{\hbar} \mathbf{p}_{+} \cdot \mathbf{q}_{-}}$, de forma que para tempos pequenos temos

$$
\begin{aligned}
& \left\langle\mathbf{q}_{+}\left|\hat{U}_{\epsilon}\right| \mathbf{q}_{-}\right\rangle \approx \frac{1}{(2 \pi \hbar)^{L / 2}} \\
& \times \int \mathrm{d} \mathbf{p}_{+} \mathrm{e}^{-\frac{i}{\hbar} H\left(\mathbf{p}_{+}, \mathbf{q}_{-}\right)} \mathrm{e}^{-\frac{i}{\hbar} \mathbf{p}_{+} \cdot\left(\mathbf{q}_{+}-\mathbf{q}_{-}\right)} .
\end{aligned}
$$

Então o propagador para o tempo completo fica

$$
\begin{aligned}
& \left\langle\mathbf{q}_{+}\left|\hat{U}_{t}\right| \mathbf{q}_{-}\right\rangle=\lim _{N \rightarrow \infty} \int \ldots \int \mathrm{d} \mathbf{q}_{N-1} \\
& \ldots \mathrm{d} \mathbf{q}_{1}\left\langle\mathbf{q}_{+}\left|\hat{U}_{\epsilon}\right| \mathbf{q}_{N-1}\right\rangle \ldots\left\langle\mathbf{q}_{1}\left|\hat{U}_{\epsilon}\right| \mathbf{q}_{-}\right\rangle \\
& =\lim _{N \rightarrow \infty} \int \frac{\mathrm{d} \mathbf{p}_{N}}{(2 \pi \hbar)^{L / 2}} \int \ldots \int \prod_{k=1}^{N-1} \frac{\mathrm{d} \mathbf{p}_{k} \mathrm{~d}_{k}}{(2 \pi \hbar)^{L}} \\
& \times \exp \left[\frac{i}{\hbar} \sum_{k=0}^{N-1}\left[\mathbf{p}_{k+1}\left(\mathbf{q}_{k+1}-\mathbf{q}_{k}\right)-\epsilon H\left(\mathbf{p}_{k+1}, \mathbf{q}_{k}\right)\right]\right]
\end{aligned}
$$

$\operatorname{com} \mathbf{q}_{N}=\mathbf{q}_{+}$e $\mathbf{q}_{0}=\mathbf{q}_{-}$.

Podemos agora reconhecer na soma do expoente deste integrando a própria ação clássica,

$$
S(\mathbf{q}+, \mathbf{q}-, t)=\int_{0}^{t} \mathrm{~d} t\left[\mathbf{p}_{t} \cdot \dot{\mathbf{q}}_{t}-H\left(\mathbf{p}_{t}, \mathbf{q}_{t}\right)\right],
$$

ao longo do caminho $\mathbf{x}_{t}=\left(\mathbf{p}_{t}, \mathbf{q}_{t}\right)$, discretizado em intervalos $\delta t=\epsilon$. O propagador assume assim a sua forma simbólica de integral de caminho:

$$
\left\langle\mathbf{q}_{+}\left|\hat{U}_{t}\right| \mathbf{q}_{-}\right\rangle=\int \mathbf{D}\left[\mathbf{x}_{t}\right] \exp \left[\frac{i}{\hbar} S\left(\mathbf{q}+, \mathbf{q}_{-}, t\right)\right] .
$$

Esta integral é análoga à de Feynman, mas os caminhos percorrem o espaço de fases livremente, sem que os momentos fiquem atrelados ao caminho das posições. Também lidamos, de saída, diretamente com a Hamiltoniana, dispensando a introdução da Lagrangiana que é mais distante da mecânica quântica. Outras construções da integral de caminho são a da representação de Weyl [9: 10 . (empregada no transporte da função de Wigner [11]), ou a de estados coerentes 12,13 (onde os caminhos percorrem um espaço de fases complexo).

A profusão de caminhos nesta bela formulação da mecânica quântica pareceriam impedir o uso direto da integral de Feynman, a não ser nos exemplos mais banais. Contudo, o princípio variacional clássico [14, 15] surge oportunamente para organizar este excesso: A ação (6) é estacionária para o caminho que coincide com a trajetória clássica. Em outras palavras, se discretizarmos os caminhos como em (5), a trajetória é definida por

$$
\frac{\partial}{\partial \mathbf{q}_{k}} S(\mathbf{q}+, \mathbf{q}-, t)=\frac{\partial}{\partial \mathbf{p}_{k}} S(\mathbf{q}+, \mathbf{q}-, t)=0
$$

para todo $k$. Como a presença dos momentos na Hamiltoniana já é quadrática, basta agora expandir o potencial entorno das posições ao longo de cada trajetória. Enumeradas pelo índice $j$, suas posições discretizadas serão $\mathbf{q}_{k}^{j}$, de modo que aproximamos

$$
V(\mathbf{q})=V\left(\mathbf{q}_{k}^{j}\right)+\left(\mathbf{q}-\mathbf{q}_{k}^{j}\right) \cdot \frac{\partial^{2}}{\partial \mathbf{q}^{2}} V\left(\mathbf{q}_{k}^{j}\right)\left(\mathbf{q}-\mathbf{q}_{k}^{j}\right)+\ldots
$$

Assim, o integrando em (5) se torna uma Gaussiana multidimensional, imediatamente integrável. Esse procedimento, conhecido como método da fase estacionária, fornece então a aproximação semiclássica do propagador,

$$
\left\langle\mathbf{q}_{+}\left|\hat{U}_{t}\right| \mathbf{q}_{-}\right\rangle_{S C}=\sum_{j} D_{j} \exp \left[\frac{i}{\hbar} S_{j}\left(\mathbf{q}_{+}, \mathbf{q}_{-}, t\right)\right]
$$

onde $S_{j}\left(\mathbf{q}_{+}, \mathbf{q}_{-}, t\right)$ é a integral da ação 6 calculada sobre a j'ésima trajetória clássica entre $\mathbf{q}_{-}$e $\mathbf{q}_{+}$. (Estamos aqui omitindo pequenas correções de Maslov [18,26].) Esta aproximação foi primeiro deduzida por van Vleck 16] já em 1928 diretamente da equação de Schrödinger.

Atingimos aqui um bom meio termo entre a mecânica clássica e a mecânica quântica: Equiparando a transformação unitária $\hat{U}_{t}$ à transformação canônica clássica, 
$C_{t}: \mathbf{x}_{-} \mapsto \mathbf{x}_{+}$, carregada pelas trajetórias hamiltonianas no tempo $t$, a ação, $S_{j}\left(\mathbf{q}_{+}, \mathbf{q}_{-}, t\right)$, toma o papel de função geratriz de $C_{t}$, por meio das equações implícitas 15, 17:

$$
\frac{\partial S_{j}}{\partial \mathbf{q}_{+}}=\mathbf{p}_{+}, \frac{\partial S_{j}}{\partial \mathbf{q}_{-}}=-\mathbf{p}_{-} .
$$

De forma mais precisa, a transformação global $C_{t}$ tem diferentes funções geratrizes na vizinhança de cada uma das trajetórias $\mathbf{x}_{t}^{j}$. A aproximação linearizada de incrementos, $C_{j}: \delta \mathbf{x}_{-} \mapsto \delta \mathbf{x}_{+}$, tomados dos pontos inicial e final de cada trajetória, será

$$
\begin{aligned}
\delta \mathbf{x}_{+} & =\left(\begin{array}{c}
\delta \mathbf{p}_{+} \\
\delta \mathbf{q}_{+}
\end{array}\right)=\left(\begin{array}{cc}
\frac{\delta \mathbf{p}_{+}}{\delta \mathbf{p}_{-}} & \frac{\delta \mathbf{p}_{+}}{\delta \mathbf{q}_{-}} \\
\frac{\delta \mathbf{q}_{+}}{\delta \mathbf{p}_{-}} & \frac{\delta \mathbf{q}_{+}}{\delta \mathbf{q}_{-}}
\end{array}\right)\left(\begin{array}{c}
\delta \mathbf{p}_{-} \\
\delta \mathbf{q}_{-}
\end{array}\right) \\
& =\mathbf{M}_{t}^{j} \delta \mathbf{x}_{-},
\end{aligned}
$$

onde a matriz Jacobiana $\mathbf{M}_{t}^{j}$ é uma matriz simplética conhecida como matriz de estabilidade, ou matriz de monodromia. A amplitude da contribuição de cada uma das trajetórias ao propagador semiclássico (10) é dada por um bloco desta matriz:

$$
\left|D_{j}\right|^{2}=\left|\operatorname{det} \frac{\partial \mathbf{q}_{+}}{\partial \mathbf{p}_{-}}\right|=\left|\operatorname{det} \frac{\partial \mathbf{p}_{-}}{\partial \mathbf{q}_{+}}\right|^{-1}=\left|\operatorname{det} \frac{\partial^{2} S_{j}}{\partial \mathbf{q}_{-} \partial \mathbf{q}_{+}}\right|^{-1} .
$$

Devemos notar que a linearização de uma transformação canônica equivale a aproximar quadraticamente a Hamiltoniana que gerou o movimento. Para toda Hamiltoniana quadrática (ainda que dependente do tempo), só existe uma única trajetória em (10), sua amplitude é constante e, melhor, essa aproximação semiclássica passa a ser exata!

A correspondência entre a mecânica clássica e a mecânica quânica é ainda mais realçada pelo foco sobre uma única característica do propagador: o traço do operador de evolução que quantifica a tendência de qualquer estado inicial de retornar sobre si mesmo:

$$
\operatorname{tr} \hat{U}_{t}=\int \mathrm{d} \mathbf{q}\left\langle\mathbf{q}\left|\hat{U}_{t}\right| \mathbf{q}\right\rangle
$$

Inserindo a aproximação semiclássica 10 para o propagador nesta expressão com $\mathbf{q}_{+}=\mathbf{q}_{-}=\mathbf{q}$ e notando que o valor estacionário do expoente,

$$
\frac{\partial S_{j}}{\partial \mathbf{q}_{+}}+\frac{\partial S_{j}}{\partial \mathbf{q}_{-}}=\mathbf{p}_{+}\left(\mathbf{q}_{+}, t\right)-\mathbf{p}_{-}\left(\mathbf{q}_{-}, t\right)=0
$$

identifica aqui o momento final, $\mathbf{p}_{+}(\mathbf{q})$ com o momento inicial $\mathbf{p}_{-}(\mathbf{q})$, deduzimos que a aproximação semiclássica para o traço, resultante do método de fase estacionária aplicado à integral (14) é

$$
\operatorname{tr}_{S C} \hat{U}_{t}=\sum_{j} D^{\prime}{ }_{j} \exp \left[\frac{i}{\hbar} S^{\prime}{ }_{j}(t)\right]
$$

(ignorando de novo pequenas correções em $\hbar$ ). Aqui temos uma soma sobre aquelas trajetórias periódicas que tiverem o período $t$ : Cada ponto de tal trajetória será um ponto fixo da transformação $C_{t}$. A ação para o retorno partindo de qualquer destes pontos será

$$
S^{\prime}{ }_{j}(t)=\oint_{j} \mathbf{p} \cdot \mathrm{d} \mathbf{q}-E_{j} t
$$

sendo $E_{j}$ a energia constante da trajetória. Em conclusão, o retorno quântico toma por apoio clássico o conjunto de trajetórias periódicas do sistema.

Quanto maior o tempo, maior o número de trajetórias periódicas de um típico sistema hamiltoniano clássico limitado. (O oscilador harmônico não é um bom exemplo!) No limite $t \rightarrow \infty$ a soma em 16 se torna infinita. Lembrando que o traço também é uma soma sobre os auto-valores $E_{n}$ da Hamiltoniana quântica,

$$
\operatorname{tr} \hat{U}_{t}=\sum_{n}\left\langle n\left|\mathrm{e}^{-i t \hat{H} / \hbar}\right| n\right\rangle=\sum_{n} \mathrm{e}^{-i t E_{n} / \hbar},
$$

obtemos

$$
\begin{aligned}
& \frac{1}{2 \pi \hbar} \int_{-\infty}^{\infty} \mathrm{d} t \mathrm{e}^{i t E / \hbar} \operatorname{tr} \hat{U}_{t} \\
& =\frac{1}{2 \pi \hbar} \sum_{n} \int_{-\infty}^{\infty} \mathrm{d} t \mathrm{e}^{i t\left(E-E_{n}\right) / \hbar} \\
& =\sum_{n} \delta\left(E-E_{n}\right) \equiv \rho(E) .
\end{aligned}
$$

Outrossim, a densidade de estados, $\rho(E)$, uma caraterística que só cabe a um sistema quântico, repousa semiclassicamente em uma soma sobre as trajetórias periódicas do sistema clássico correspondente. No entanto, devemos tomar um cuidado: Não basta inserir 16 em 19 para calcular a aproximação semiclássica da densidade de estados. Perderíamos então a forte contribuição das trajetórias que não saem do lugar, no entorno de $t=0$, ou seja, todos os pontos iniciais. O cálculo deste termo de Weyl fornece uma interpolação suave, $\bar{\rho}(E)$, para os sucessivos picos da densidade de estados, mas que não cabe tratar aqui. É o termo que sobra em $\rho(E)=\bar{\rho}(E)+\tilde{\rho}(E)$ que vai somando contribuições cada vez mais oscilatórias das trajetórias periódicas de crescente período, cuja interferência em (16) vai afinando os picos finos de 19 .

A condição estacionária para a integral do lado esquerdo de 19 é

$$
\frac{\partial}{\partial t}\left(S^{\prime}{ }_{j}+E t\right)=E-E_{j}=0,
$$

ou seja, ela seleciona as órbitas periódicas (curvas fechadas sem diferenciação de tempo) sobre a camada de energia $H(\mathbf{x})=E$, com a ação reduzida

$$
\sigma_{j}(E)=\oint_{j} \mathbf{p} \cdot \mathrm{d} \mathbf{q} .
$$

Assim, a componente oscilatória da densidade de estados tem a aproximação semiclássica

$$
\tilde{\rho}_{S C}(E)=\sum_{j} D_{j}^{\prime \prime} \exp \left[\frac{i}{\hbar} \sigma_{j}(E)\right],
$$


conhecida como a fórmula do traço de Gutzwiller (vide [18 e também Balian e Bloch [19]).

A beleza desta construção formal não deve dissipar sérias dúvidas sobre seu real significado: É como se surgisse um contexto onde o quântico fosse o conjugado de Fourier do clássico! Será que podemos realmente resolver os picos finos e possíveis degenerescências da densidade de estados ao incluir cada vez mais órbitas periódicas? Enfim, a soma sobre elas converge? O teorema de recorrência de Poincaré 20.21 garante que, em qualquer vizinhança de um ponto na camada de energia, haverá uma trajetória que eventualmente retorna. Partindo desta, é fácil encontrar uma órbita periódica adjacente. Então vemos que elas são densas sobre o espaço de fases, sendo, entretanto, infinitamente raras: A probabilidade de um ponto inicial arbitrário cair numa órbita peródica é nula. Como que esse especialíssimo conjunto das órbitas mais simples pode tipificar a rica gama de movimentos clássicos que abrangem do ultra regular (movimento harmônico multidimensional), ao integrável (trajetórias vinculadas a superfícies invariantes de dimensão menor que a camada de energia), aos movimentos predominantemente caóticos, caracterizados pelo afastamento exponencial de trajetórias vizinhas? Em contraste com as trajetórias periódicas, uma trajetória típica de um sistema caótico não fecha nunca e, com o tempo, percorre ergodicamente todo espaço de fases disponível energeticamente. Será que, mesmo assim, podemos nos valer da fórmula do traço para explorar a maneira que esta rica variedade clássica venha a afetar propriedades mensuráveis dos sistemas quânticos correspondentes? Não é um empreendimento fácil, pois a linearidade da mecânica quântica impede uma correspondência direta com as consequências da não linearidade clássica.

$\mathrm{Na}$ contramão de todas essas dúvidas existe um emprego notável da fórmula do traço como organizador do espectro de energias. A densidade de estados de um sistema que é classicamente caótico apresenta uma cacofonia de níveis. Contudo, sua transformada de Fourier apenas devolve, de acordo com 19 , o tr $\hat{U}_{t}$ de onde partimos. Fora o pico em $t=0$, haverá um pico em $t_{1}$, a primeira órbita periódica, seguido por picos em $t_{j}$, para cada órbita periódica subsequente. O espectro de energia na parte superior da figura 1 apresenta o espectro de energia experimental (ressonâncias espectrais) do hidrogênio altamente excitado em um campo magnético 22 . Sem o campo magnético teríamos os níveis do átomo de Rydberg (integrável) organizados por seus momentos angulares. A quebra de simetria pelo campo destrói as superfícies invariantes clássicas: Os únicos invariantes são as órbitas periódicas isoladas. Por outro lado, o campo desorganiza os níveis quânticos. Todos os estados compartilham características de ergodicidade quântica [23 24], mas são mutuamente ortogonais, apesar de não serem distinguíveis por nenhuma simetria. Uma simples transformada de Fourier reimpõe uma nova ordem, os picos nítidos de tr $\hat{U}_{t}$, que

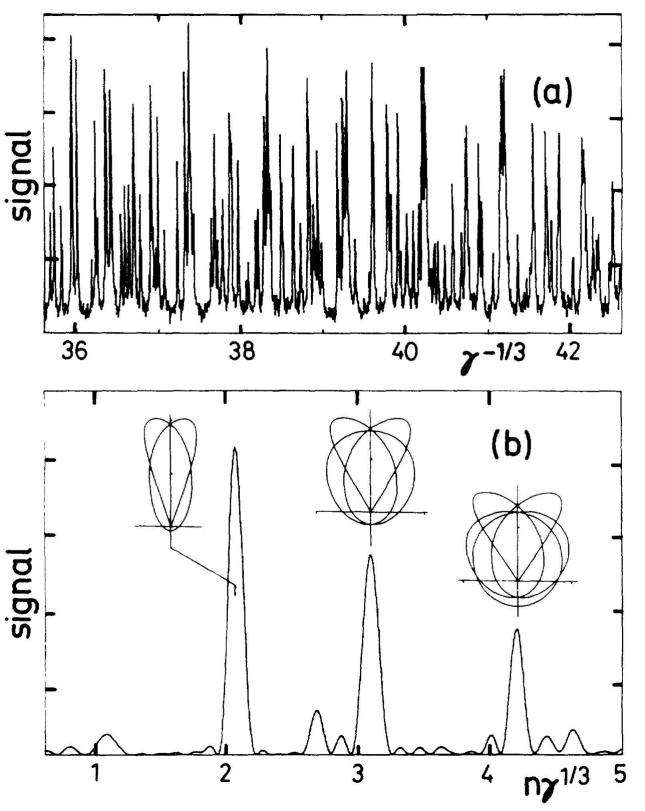

Figura 1: (a) Ressonâncias espectrais de um átomo de hidrogênio em um campo magnético. (b) Transformada de Fourier do espectro, com esboço das órbitas periódicas cujo período correspondente a cada pico.

são mostrados junto com o esboço das órbitas periódicas que lhes dão origem.

No domínio do tempo não existe o problema de convergência. Só quando passamos para o domínio de energia é que surgem as dificuldades. De fato, em geral a fórmula do traço não converge, mas isso não impede seu uso como ferramenta exploratória da correspondência clássico-quântica. A diferença entre os sistemas clássicos regulares e os caóticos se reflete duplamente no traço de Gutzwiller. Primeiro, pode-se deduzir que o numero de órbitas periódicas cresce exponencialmente com o seu período, $\tau_{j}$, se o movimento for caótico [25,26], enquanto que este crescimento é muito mais lento para sistemas regulares. Em contrapartida, a amplitude da contribuição de cada órbita do sistema caótico cai exponencialmente com $\tau_{j}$. De fato, a intensidade $\left|D^{\prime \prime}{ }_{j}\right|^{2} \cong \mathrm{e}^{-\lambda \tau_{j}}$, onde $\lambda$ é o expoente de Lyapunov que caracteriza o afastamento médio das trajetórias vizinhas do movimento caótico. Assim, a maneira que uma única tragetória caótica varre densamente toda a camada de energia se reflete na densa população de órbitas periódicas de período arbitrariamente longo.

A combinação destas duas características permite avaliar as correlações do espectro para pequenos intervalos, $\Delta$, de energia,

$$
\begin{aligned}
& K(\Delta) \equiv W^{-1} \int \mathrm{d} E \tilde{\rho}(E) \tilde{\rho}(E+\Delta) \\
& \approx \Re \sum_{j l} \int \mathrm{d} E D^{\prime \prime}{ }_{j}(E) D^{\prime \prime}{ }_{l}(E) \\
& \times \exp \left[\frac{i}{\hbar}\left[\sigma_{j}(E)-\sigma_{l}(E+\Delta)\right]\right],
\end{aligned}
$$


sendo a janela de integração, $W$, clássicamente pequena, porém ainda contendo muitos níveis quânticos. O fato de $\mathrm{d} \sigma_{j} / \mathrm{d} E=\tau_{j}(E)$, o período da órbita, indica que a maioria desses integrandos são altamente oscilatórios. Conservando apenas os termos em que $j=l$, obtemos então a aproximação diagonal,

$$
K_{d}(\Delta)=\Re \sum_{j}\left[D^{\prime \prime}{ }_{j}(E)\right]^{2} \exp \left[\frac{i}{\hbar} \tau_{j}(E) \Delta\right] .
$$

É notável que a indiscutível crueza deste passo ainda preserva a informação essencial da correlação da densidade de estados: Sua transformada de Fourier (devidamente normalizada) concorda essencialmente com a das correlações universais dos ensembles de matrizes aleatórias 25, 26, 28. Assim, atingimos uma explicação física para a conjectura de Bohigas, Giannoni e Schmit [27], de base empírica, que as correlações do espectro de energias dos sistemas caóticos coincidem com esses espectros universais, fazendo até a distinção entre as classes de universalidade das matrizes aleatórias, como a presença ou ausência de simetria de reversão temporal.

Será que a aproximação diagonal é o máximo que podemos almejar? A profusão de órbitas periódicas de um sistema caótico parece inibir qualquer pretensão de contabilizar as contribuições dos pares de órbitas em (23). Mais uma aplicação surpreendente do traço de Gutzwiller foi então a de partir retroativamente dos desvios das correlações semiclássicas relativamente às correlações universais e conjecturar daí a existência de pares de trajetórias, com quase a mesma ação, levando a termos quase diagonais a serem adicionados a $K_{d}(\Delta)$. Parece muita pretensão desencavar uma novidade na estrutura de órbitas periódicas clássicas, investigadas desde Poincaré, com base em aproximações semiclássicas que passam por cima de qualquer cuidado com convergência. Não obstante, Sieber e Richter mostraram que existem sempre pares para as órbitas que traçam figuras de oito, como mostradas na figura 2 e que estas fornecem a contribuição justa para aprimorar a concordância das correlações semiclássicas com as correlações universais 29 . Assim, a ponte clássico-quântica abarca trânsito nos dois sentidos.

Enfim, ainda há muito a entender sobre essa correspondência básica. A experiência indica que mais vale levar bem em conta a estrutura geral de ambas essas

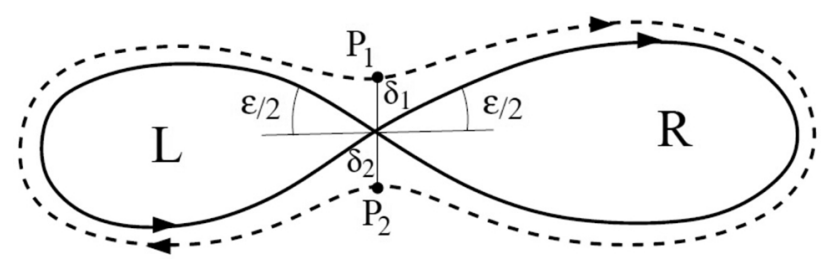

Figura 2: As órbitas periódicas nunca se auto-intersectam no espaço de fases, mas sua projeção nas posições pode formar uma 'figura de oito'. Na vizinhança dessas, existe sempre uma órbita periódica vizinha com quase a mesma ação. maravilhosas teorias do que fazer um grande esforço de estender para ordens superiores as aproximações semiclássicas, onde elas acabam perdendo seu conteúdo físico. A natureza dos auto-estados de uma Hamiltoniana caótica continua a ser um grande desafio. A bem da verdade, os estados que hoje em dia se preparam e manipulam no laboratório, como os átomos de Rydberg, não são deste tipo exótico, sendo distinguidos por suas simetrias. Como pescar um estado ergódico no mar de nívies da figura 1? Quais seriam seus recursos quânticos? Talvez seja o caso de considerá-los por hora como uma reserva natural de estados quânticos, ainda não tocados pelo homem, tanto mais nobres na sua inacessibilidade.

Devemos lembrar que a verdadeira dinâmica quântica se desenvolve no domínio do tempo. Não queremos só avaliar o espectro de energias; lidamos com a evolução temporal de outros personagens como observáveis, seu valor esperado, suas correlações e aí podemos, ao medir, fazer uma análize espectral. As aproximações semiclássicas fornecem expressões para todos esses elementos em termos do conjunto completo de trajetórias clássicas [30]. Para um tempo finito, não precisamos nos preocupar com a natureza regular ou caótica da evolução clássica. Mesmo para sistemas abertos, de evolução não unitária, desenvolvemos expressões semiclássicas [31] dentro de aproximações markovianas [1] e, talvez, se venha a encontrar novas extensões do método de Feynman-Vernon para alargar ainda mais a ponte entre a mecânica clássica e a mecânica quântica.

\section{Agradecimentos}

Agradeço os comentários críticos de Mucio Continentino e Gabriel Lando, assim como apoio finceiro do CNPq e do Instituto Nacional de informação Quântica.

\section{Referências}

[1] D. Giulini, E. Joos, C. Kiefer, J. Kupsch, I.-O. Stamatescu and H.D. Zeh, Decoherence and the Appearance of a Classical World in Quantum Theory (Springer, Berlin, 1996).

[2] W. Zurek, Rev. Mod. Phys. 75, 715 (2003).

[3] R.P. Feynman and F.L. Vernon, Ann. Phys. 24, 118 (1963).

[4] A.O. Caldeira and A.J. Leggett, Ann. Phys. (NY) 149, 374 (1983).

[5] P.A.M. Dirac, Principles of Quantum Mechanics (Oxford University Press, Oxford, 1947).

[6] R.P. Feynman, Rev. Mod. Phys. 20, 367 (1948).

[7] R.P. Feynman, Statistical Mechanics: A Set of Lectures (Benjamin, Menlo Parrk, 1972).

[8] M.S. Marinov, Phys. Reports 60, 1 (1960).

[9] A.M. Ozorio de Almeida, Proc. R. Soc. Lond. A 439, 139 (1992).

[10] A.M. Ozorio de Almeida, Phys. Rep. 295, 265 (1998).

[11] E.P. Wigner, Phys Rev 40, 749 (1932). 
[12] J.R. Klauder and B.S. Skagerstam, Coherent States, Applications in Physics and Mathematical Physics (World Scientific, Singapore, 1985).

[13] M. Baranger, M.A.M. de Aguiar, F. Keck, H.J. Korsch and B. Schellas, J. Phys. A: Math. Gen. 34, 7227 (2001).

[14] C. Lanczos, The Variational Principles of Mechanics (Dover Publications, New York, 1970).

[15] V.I. Arnold, Mathematical Methods of Classical Mechanics (Springer, Berlin, 1978).

[16] J.H. van Vleck, Proc. Nac. Acad. Sci. 14, 178 (1928).

[17] H. Goldstein, Classical Mechanics (Addison-Wesley, Reading, 1980), 2nd ed.

[18] M. Gutzwiller, Chaos in Classical and Quantum Mechanics (Springer-Verlag, New York, 1990).

[19] R. Balian and C. Bloch, Ann. Phys. NY 85, 514 (1974).

[20] R. Mañé, Teoria Ergódica (IMPA, Rio de Janeiro, 1983).

[21] O.E. Lanford, em Chaotic Behaviour of Deterministic Systems, editado por G. Ioss, R.H.G Helleman and R. Stora (Les Houches 36, Amsterdam, 1983).

[22] A. Holle, J. Main, G. Wiebusch, H. Rottke and K.H. Welge, Phys. Rev. Lett. 61, 161 (1988).

[23] A. Shnirelman, Uspekhi Mat. Nauk. 29, 181 (1974).

[24] Y. Colin de Verdière, Comm. Math. Phys. 102, 497 (1985).

[25] J.H. Hannay and A.M. Ozorio de Almeida, J. Phys. A 17, 3429 (1984).

[26] A.M. Ozorio de Almeida, Hamiltonian Systems: Chaos and Quantization (Cambridge University Press, Cambridge, 1988).

[27] O. Bohigas, M.J. Giannoni and C. Schmidt, Phys. Rev. Lett. 52, 1 (1984).

[28] M.V. Berry, Proc. R. Soc. Lond. A 400, 229 (1985).

[29] M. Sieber and K. Richter, Physica Scripta 2001, 128 (2001).

[30] A.M. Ozorio de Almeida and O. Brodier, J. Phys. A 49, $185302(2016)$

[31] A.M. Ozorio de Almeida and O. Brodier, Phil. Trans. R. Soc. A 369, 260 (2011). 